\title{
Determining Inelastic Mean Free Path by Electron Energy Loss Spectroscopy
}

\author{
Qiang Jin and Du Li
}

Micron Technology, Inc., Boise, ID 83707, USA

Among the ways of measuring TEM foil thickness, the log-ratio method [1] based on electron energy-loss spectroscopy (EELS) is quick and less limited. Its accuracy, however, mainly depends on how accurately the inelastic mean free path $(\lambda)$ can be determined. By using the method proposed by Jin [2], the values of $\lambda$ for a variety of materials have been accurately measured in this work by depositing a thin amorphous film of the material on a (001) Si wafer. Fig. 1a shows one of the cross-sectional TEM micrographs of the deposited films. TEM specimens used for $\lambda$ measurement were made wedge-shaped in plan-view, consisting of three layers including an amorphous damage layer caused by sample preparation (Fig. 1b). The ratios of thickness (t) to $\lambda$ for each layer are related to EELS intensities by [2]:

$$
\ln \left(\mathrm{I}_{\mathrm{T}} / \mathrm{I}_{0}\right)=\mathrm{t}_{\mathrm{c}} / \lambda_{\mathrm{c}}+\mathrm{t}_{\mathrm{f}} / \lambda_{\mathrm{f}}+\mathrm{t}_{\mathrm{a}} / \lambda_{\mathrm{a}}
$$

where $\mathrm{I}_{\mathrm{T}}$ and $\mathrm{I}_{0}$ are the total intensity of the EEL spectrum and the intensity of the zero-loss peak. The subscripts $c, f$ and $a$ are notations of the crystalline $\mathrm{Si}$, the deposited film, and the damage layer, respectively. To determine the mean free path of the deposited amorphous film $\lambda_{\mathrm{f}}, \mathrm{I}_{\mathrm{T}}, \mathrm{I}_{0}$ and $\mathrm{t}_{\mathrm{c}}$ are measured for different locations. Then $\ln \left(\mathrm{I}_{\mathrm{T}} / \mathrm{I}_{0}\right)$ versus $\mathrm{t}_{\mathrm{c}}$ is plotted. The crystalline thickness $\mathrm{t}_{\mathrm{c}}$ was measured by the convergent beam electron diffraction (CBED) method [3]. As shown in Fig. 2, the best-fit lines of the experimental data for different films are parallel to each other with the slope corresponding to the reciprocal of the mean free path of $\mathrm{Si}$, i.e., $1 / \lambda_{\mathrm{c}}$. Assuming crystalline and amorphous Si have similar mean free paths, i.e., $\lambda_{c}=\lambda_{a}, \mathrm{t}_{\mathrm{a}}$ is then obtained from the intercept of the best-fit line of the Si foil without a deposited amorphous film. For grind-back sample followed by final cleaning in an ion mill, $\mathrm{t}_{\mathrm{a}}$ is about $6 \mathrm{~nm}$, which was verified by direct TEM measurement. In addition, $t_{f}$ was measured by cross-sectional TEM, as shown in Fig. 1a. Thus, the mean free paths of the deposited amorphous films are obtained from the intercepts of the best-fit lines, which correspond to $\left(t_{f} / \lambda_{f}+t_{a} / \lambda_{a}\right)$. The measured values for different materials are listed in Table 1 , in which the measured values for crystalline $\mathrm{Al}, \mathrm{Si}$, and $\mathrm{Ag}$ foils are also included.

The measured values of $\lambda$ were compared with those calculated based on the parameterization formula developed by Malis et al [1] and differences as large as $47 \%$ were found for some materials (Fig.3), though mostly within 20\%. One factor in the formula is the average energy $\operatorname{loss} \mathrm{E}_{\mathrm{m}}=7.6 \mathrm{Z}^{0.36}$, where $\mathrm{Z}$ is the effective atomic number [1]. This term was obtained based on an atomic model without the consideration of the solid-state effects. With this consideration, we propose to revise the original term to $\mathrm{E}_{\mathrm{m}}=42.5 \mathrm{Z}^{0.47} \rho / \mathrm{A}$, where $\mathrm{A}$ is the average atomic weight and $\rho$ is the density. For deposited films, $\rho$ was determined by weighing the wafer precisely before and after deposition, then dividing the film weight by its volume. With the modification of this factor, differences between mean free paths determined experimentally and those calculated from the formula are less than $6 \%$. 


\section{References}

[1] T. Malis, S.C. Cheng, and R.F. Egerton, J. Elec. Microsc. Tech. 8 (1988) 193.

[2] Q. Jin, Microsc. Microanal. 10, Suppl. 2 (2004) 882.

[3] S.M. Allen, Phil. Mag. A43 (1981) 325.
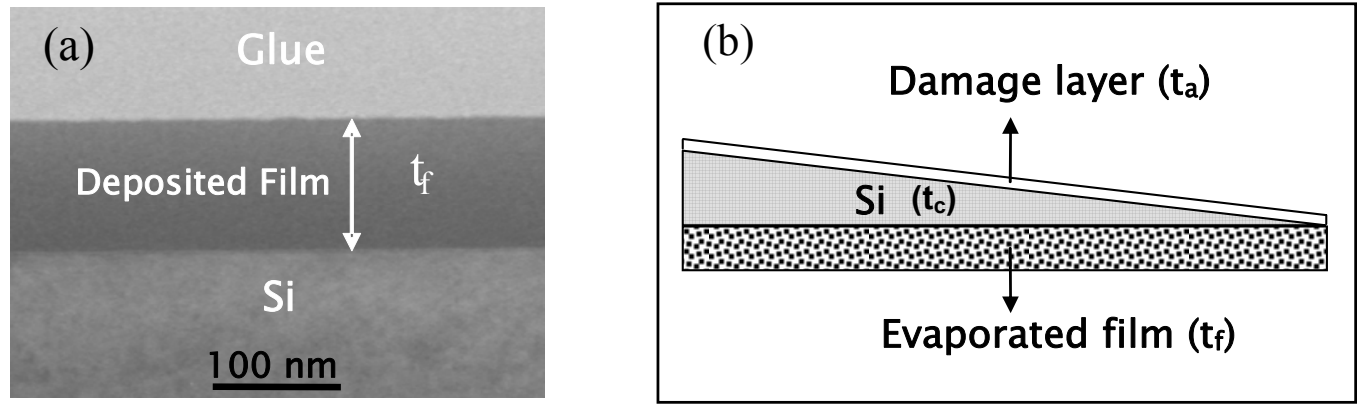

Fig. 1 (a) TEM micrograph of a representing deposition film on Si; (b) Schematic of a TEM specimen used for mean free path measurement, showing that it consists of three layers.

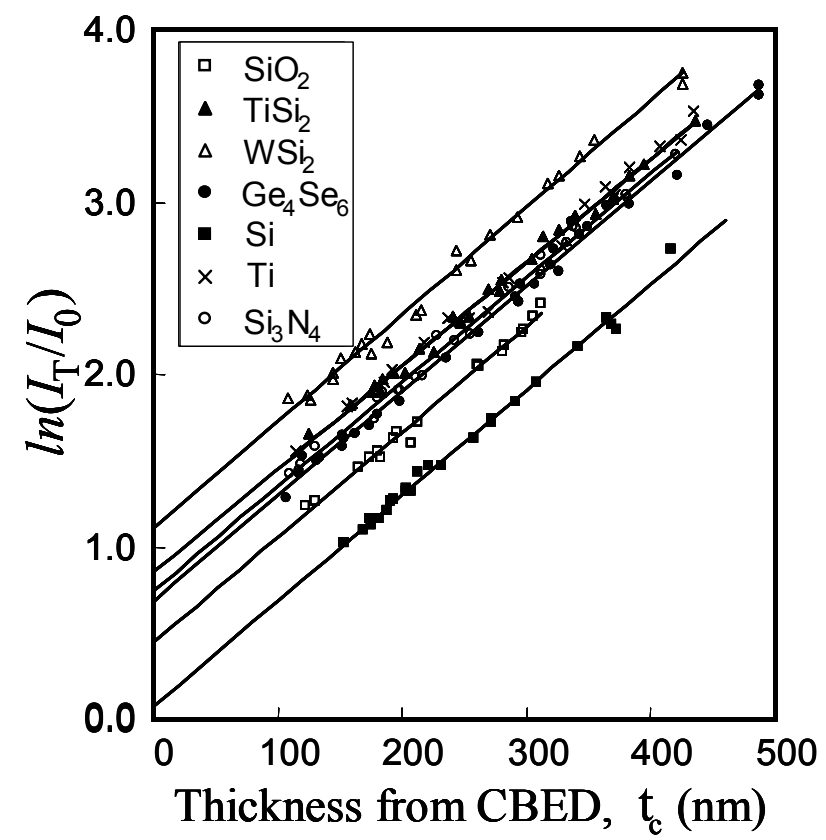

Fig. $2 \ln \left(\mathrm{I}_{\mathrm{T}} / \mathrm{I}_{0}\right)$ versus $\mathrm{t}_{\mathrm{c}}$ for the evaporated films and the Si foil without deposition

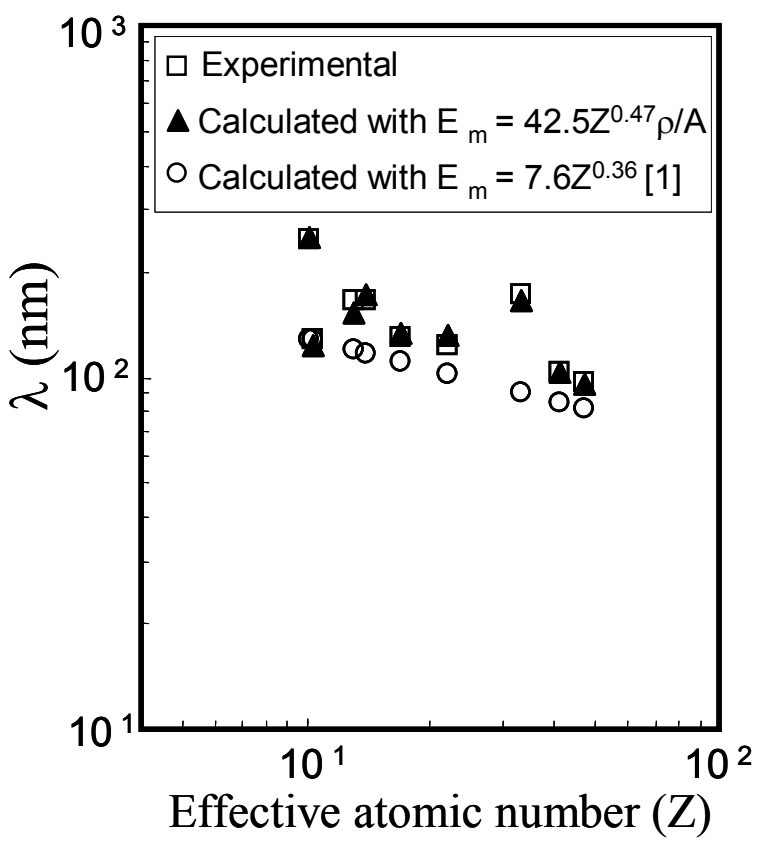

Fig. 3 Comparison of experiments with calculations in log-log plot of $\lambda$ versus $Z$.

Table 1 The values of $\lambda$ measured in TEM image mode with convergence angle $\alpha=10 \mathrm{mrad}$, collection angle $\beta=20 \mathrm{mrad}$ and incident electron energy $\mathrm{E}_{0}=200 \mathrm{keV}$.

\begin{tabular}{lccccccccc}
\hline Materials & $\mathrm{Si}$ & $\mathrm{Al}$ & $\mathrm{Ag}$ & $\mathrm{Ti}$ & $\mathrm{TiSi}_{2}$ & $\mathrm{WSi}_{2}$ & $\mathrm{SiO}_{2}$ & $\mathrm{Si}_{3} \mathrm{~N}_{4}$ & $\mathrm{Ge}_{4} \mathrm{Se}_{6}$ \\
\hline$\lambda(\mathrm{nm})$ & 164 & 164 & 97 & 123 & 130 & 103 & 245 & 128 & 172 \\
\hline
\end{tabular}

